\title{
Artificial Intelligence in Neuroscience and Systems Biology: Lessons Learnt, Open Problems, and the Road Ahead
}

\author{
Daniel Berrar, ${ }^{1,2}$ Naoyuki Sato, ${ }^{3}$ and Alfons Schuster ${ }^{4,5}$ \\ ${ }^{1}$ Systems Biology Research Group, Centre for Molecular Biosciences, School of Biomedical Sciences, University of Ulster, Cromore Road, \\ BT52 1SA, Coleraine, Northern Ireland \\ ${ }^{2}$ Systems Biology Department, Cancer Institute, Japanese Foundation for Cancer Research, Tokyo, Japan \\ ${ }^{3}$ Department of Complex Systems, Future University Hakodate, 116-2 Kamedanakano-cho, Hakodate, Hokkaido 041-8655, Japan \\ ${ }^{4}$ School of Computing and Mathematics, Faculty of Computing and Engineering, University of Ulster, Shore Road, New-Townabbey, \\ Co. Antrim, BT37 OQB, Northern Ireland \\ ${ }^{5}$ Laboratory for Dynamics of Emergent Intelligence, RIKEN Brain Science Institute, Wako-shi, Saitama 351-0198, Japan
}

Correspondence should be addressed to Daniel Berrar, dp.berrar@ulster.ac.uk

Received 31 January 2010; Accepted 31 January 2010

Copyright (C) 2010 Daniel Berrar et al. This is an open access article distributed under the Creative Commons Attribution License, which permits unrestricted use, distribution, and reproduction in any medium, provided the original work is properly cited.

"We can only see a short distance ahead, but we can see plenty there that needs to be done."

Alan M. Turing

Since its conception in the mid 1950s, artificial intelligence with its great ambition to understand intelligence, its origin, and creation, in natural and artificial environments alike, has been a truly multidisciplinary field that reaches out and is inspired by a great diversity of other fields in perpetual motion. Rapid advances in research and technology in various fields have created environments into which artificial intelligence could embed itself naturally and comfortably. Neuroscience with its desire to understand nervous systems of biological organisms and system biology with its longing to comprehend, holistically, the multitude of complex interactions in biological systems are two such fields. They target ideals artificial intelligence has dreamt about for a long time including the computer simulation of an entire biological brain or the creation of new life forms from manipulations on cellular and genetic information in the laboratory.

The scope for artificial intelligence, neuroscience, and computational systems biology is extremely wide. The motivation of this special issue is to create a bird-eye view on areas and challenges where these fields overlap in their defining ambitions and where these fields may benefit from a synergetic mutual exchange of ideas. The rationale behind this special issue is that a multidisciplinary approach in modern artificial intelligence, neuroscience, and systems biology is essential and that progress in these fields requires a multitude of views and contributions from a wide spectrum of contributors. This special issue, therefore, aims to create a centre of gravity pulling together academic researchers and industry practitioners from a variety of areas and backgrounds to share results of current research and development and to discuss existing and emerging theoretical and practical problems in artificial intelligence, neuroscience, and systems biology transporting them beyond the event horizon of their individual domains.

If the contributions in this special issue are to be classified (crudely) according to their main thrust, then the articles close to the neuroscience camp are devoted to the themes of (i) the characterization of in vitro neuronal networks cultured on multielectrode array (MEA) chips, (ii) the simulation of human episodic memory by using a computational model of the hippocampus, and (iii) information processing in natural and artificial olfactory systems. Novellino and Zaldívar propose a combination of recurrence quantification analysis based on recurrence plots and conventional statistical analysis for neuronal electrophysiology. They investigate their approach by studying the variation of spontaneous electrophysiological activity of in vitro neuronal networks on multielectrode array chips. Sato and Yamaguchi review computational models of the hippocampus and discuss their own computational model of human episodic memory based on neural synchronization. Using computer simulations and human eye movement data, they demonstrate the validity of their model to predict human memory recall. From 
an evolutionary perspective, Stafford reviews the biological constraints of the physical properties of neurons and the implication for the construction of artificial neural networks. van der Velde discusses fundamentals of human cognitive processes and proposes models for grounded architectures of cognition. Makino and Yano investigate olfaction as a relatively simple biological information processing system and report their computational works with a focus on the temporal dimension.

The article by Browne et al. reviews computational techniques to infer protein-protein interaction networks, which may help decipher novel drug targets.

Two articles are supported by a strong background in artificial intelligence and focus on learning algorithms. Schuster and Yamaguchi investigate game theoretic concepts and present a novel learning algorithm for a paired neuron system. Sridharan reports on novel bootstrapped learning techniques to process visual inputs that allow a mobile robot to autonomously adapt its behavior to illumination changes.

In summary, this special issue informs the research community at large about an exciting and stimulating relationship between artificial intelligence, neuroscience, and systems biology. The special issues provides access to many state-of-the-art theoretical and applied problems in these hugely exciting fields that are so relevant for modern science. This special issue is also intended as a platform to bridge cultural and technological gaps between these disciplines. Ultimately, the contributions in this special issue should convey to its readership the enthusiasm the editors and authors of this issue share for their respective fields.

Daniel Berrar

Naoyuki Sato

Alfons Schuster 

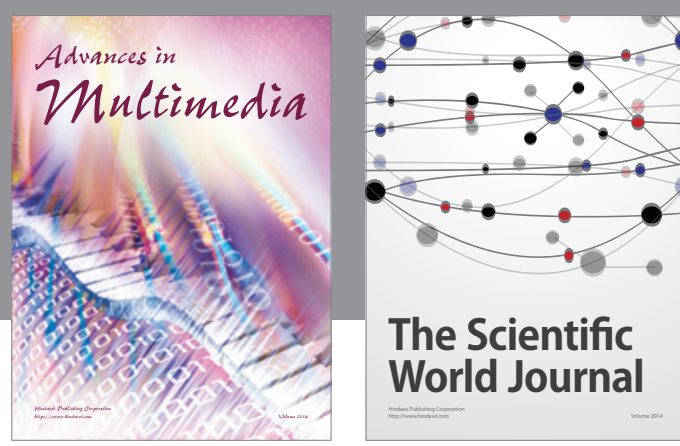

The Scientific World Journal
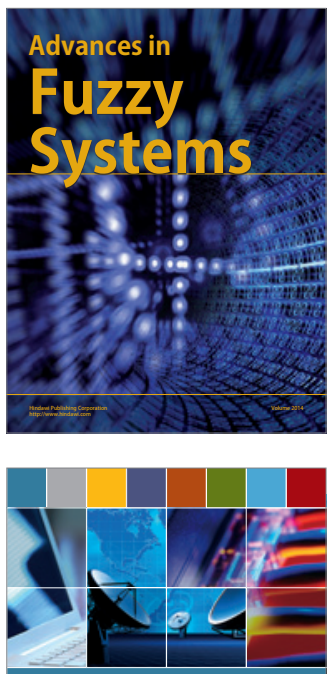

Computer Networks and Communications
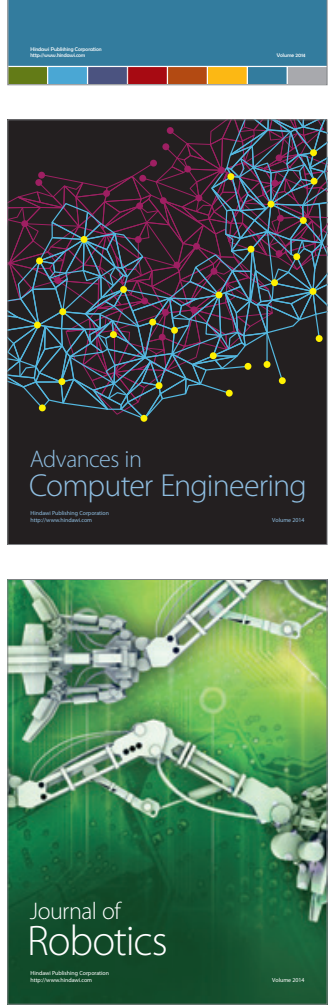
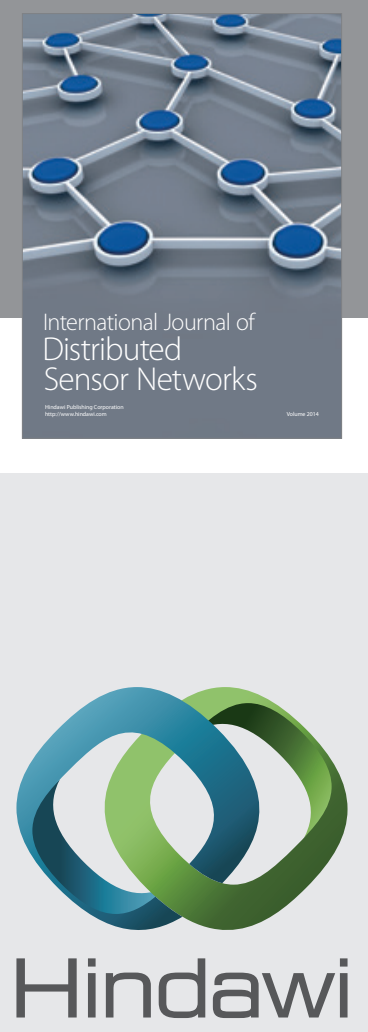

Submit your manuscripts at

http://www.hindawi.com
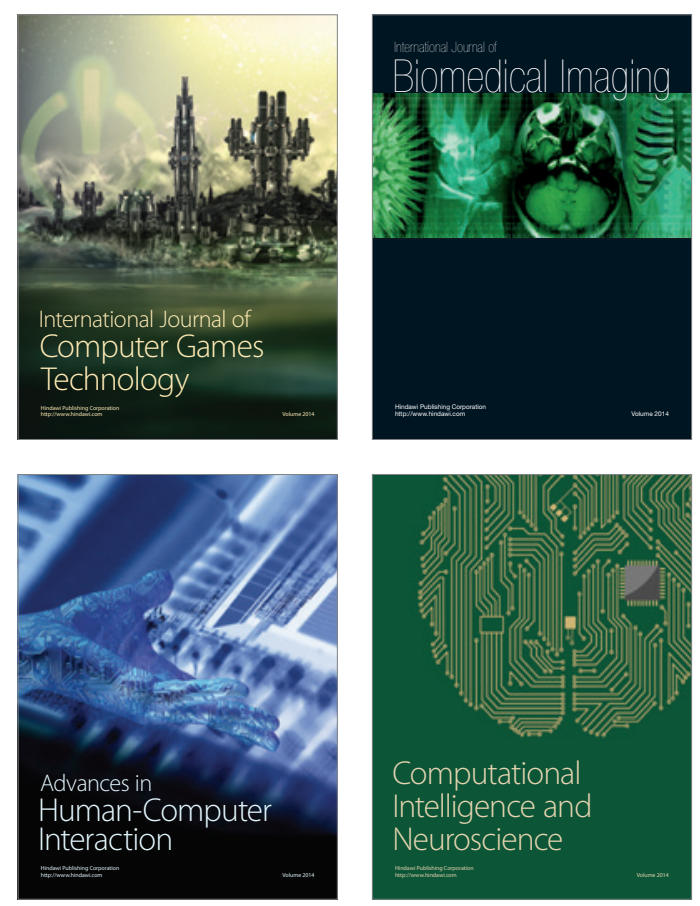
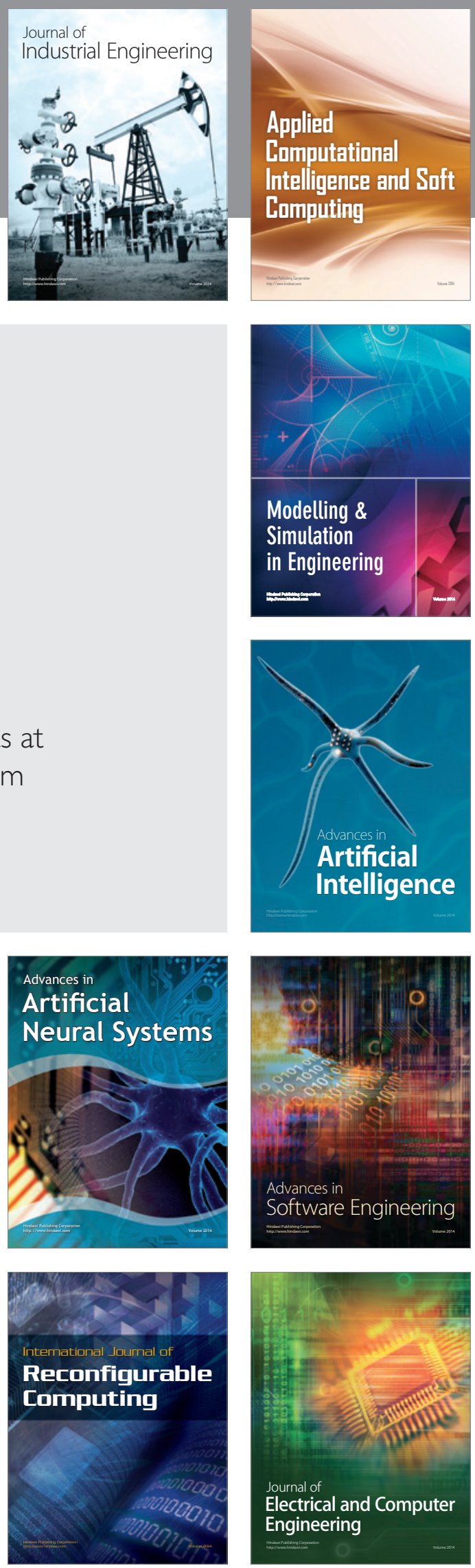